\title{
Emerging roles for macrophages in cardiac injury: cytoprotection, repair, and regeneration
}

\author{
Nikolaos G. Frangogiannis \\ Wilf Family Cardiovascular Research Institute, Department of Medicine (Cardiology), Albert Einstein College of Medicine, New York, New York, USA.
}

\begin{abstract}
The mammalian heart contains a population of resident macrophages that expands in response to myocardial infarction through the recruitment of monocytes. Infarct macrophages exhibit high phenotypic diversity and respond to microenvironmental cues by altering their functional properties and secretory profile. In this issue of the $J C I$, de Couto and colleagues demonstrate that infiltrating macrophages can be primed to acquire a cardioprotective phenotype in ischemic heart and exert this proactive effect through activation of an antiapoptotic program in cardiomyocytes. This study supports the growing body of evidence that suggests that macrophage subpopulations can be modulated to mediate cytoprotective, reparative, and even regenerative functions in the infarcted heart. The cellular mechanisms and molecular signals driving these macrophage phenotypes are yet unknown; however, harnessing the remarkable potential of the macrophage in regulating cell survival and tissue regeneration may hold therapeutic promise for myocardial infarction.
\end{abstract}

\section{Cardiac macrophages: resident} and recruited by damage

The adult mammalian heart contains a relatively small population of resident macrophages that may participate in immunosurveillance of myocardial tissue (1-3). Following myocardial infarction, the cardiac macrophage population is markedly expanded through the recruitment of abundant mononuclear cells $(1,2,4)$ that differentiate into macrophages (5). Traditionally, infarct macrophages are viewed as phagocytotic cells that clear dead cells and matrix debris from the wound and as regulators of inflammation, fibrosis, and angiogenesis. In the dynamic environment of the infarct, macrophages can acquire a wide range of phenotypes. While macrophages have been shown to affect cardiomyocyte function and survival in vitro (6, $7)$, the potential role(s) of these cells in modulating cardiomyocyte responses in vivo remains poorly understood.
Over the last ten years, members of the Marbán group have pioneered the use of cardiosphere-derived cells (CDCs), a population of cardiac-derived cells with reparative and regenerative functions, as cell therapy for myocardial infarction (8). In experimental animal models of myocardial infarction, administration of CDCs results in a wide range of beneficial actions, including enhanced regeneration, improved function, attenuation of adverse remodeling, and reduced infarct size; however, it is not clear how CDCs exert their protective effect. In this issue, de Couto and coworkers (9) report that infarct macrophages can acquire cardioprotective phenotypes that promote survival of ischemic cardiomyocytes and that these protective effects are conferred by CDCs. Specifically, CDCs induced a unique, protective macrophage phenotype that attenuates oxidative stress-mediated cardiomyocyte apoptosis. Moreover, therapy with CDC-

Related Article: p. 3147

Conflict of interest: The author has declared that no conflict of interest exists.

Reference information: J Clin Invest. 2015;125(8):2927-2930. doi:10.1172/JCI83191.

primed macrophages alone recapitulated the beneficial actions of the CDCs on the ischemic and reperfused heart. These findings add to a growing body of recent in vivo evidence that suggests that macrophages exert protective actions on the injured heart $(10,11)$.

\section{Macrophages regulate cardiomyocyte survival in ischemic myocardium}

Myocardial ischemia activates a wave of ischemic death that moves as a front and is characterized by transmural progression of cellular necrosis from the more vulnerable subendocardial areas to the subepicardium, as the duration of coronary occlusion increases (12). The susceptibility of cardiomyocytes to ischemic death is dependent on several microenvironmental factors. Cardiomyocytes are enmeshed into an extracellular matrix network and are often in close contact with interstitial cells, including fibroblasts, vascular cells, and macrophages. While extracellular matrix proteins transduce key prosurvival signals that may prevent cardiomyocyte apoptosis in the ischemic environment, interstitial, noncardiomyocytes may secrete cytokines, growth factors, and proteases that critically regulate cardiomyocyte survival. De Couto and coworkers provide in vivo evidence that suggests that a unique subpopulation of CDC-primed macrophages activates antiapoptotic pathways and is thereby capable of protecting cardiomyocytes from ischemic death (9). Although de Couto and colleagues did not dissect the molecular signals that are required for macrophage-mediated cardioprotection, several distinct pathways may be involved (Figure 1). First, CDC-modulated macrophages may secrete factors with antiapoptotic actions. A recent study identified infarct monocytes and macrophages as an important source of myeloid-derived growth factor (MYDGF), a secreted protein that promotes cardiomyocyte survival (13). In addition, release of IL-10, FGF-1, IGF-1, 


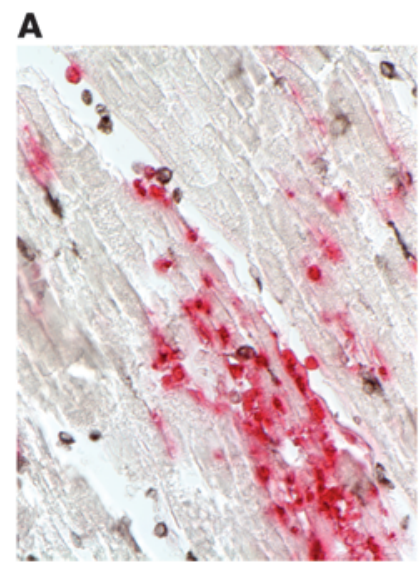

$\mathbf{B}$
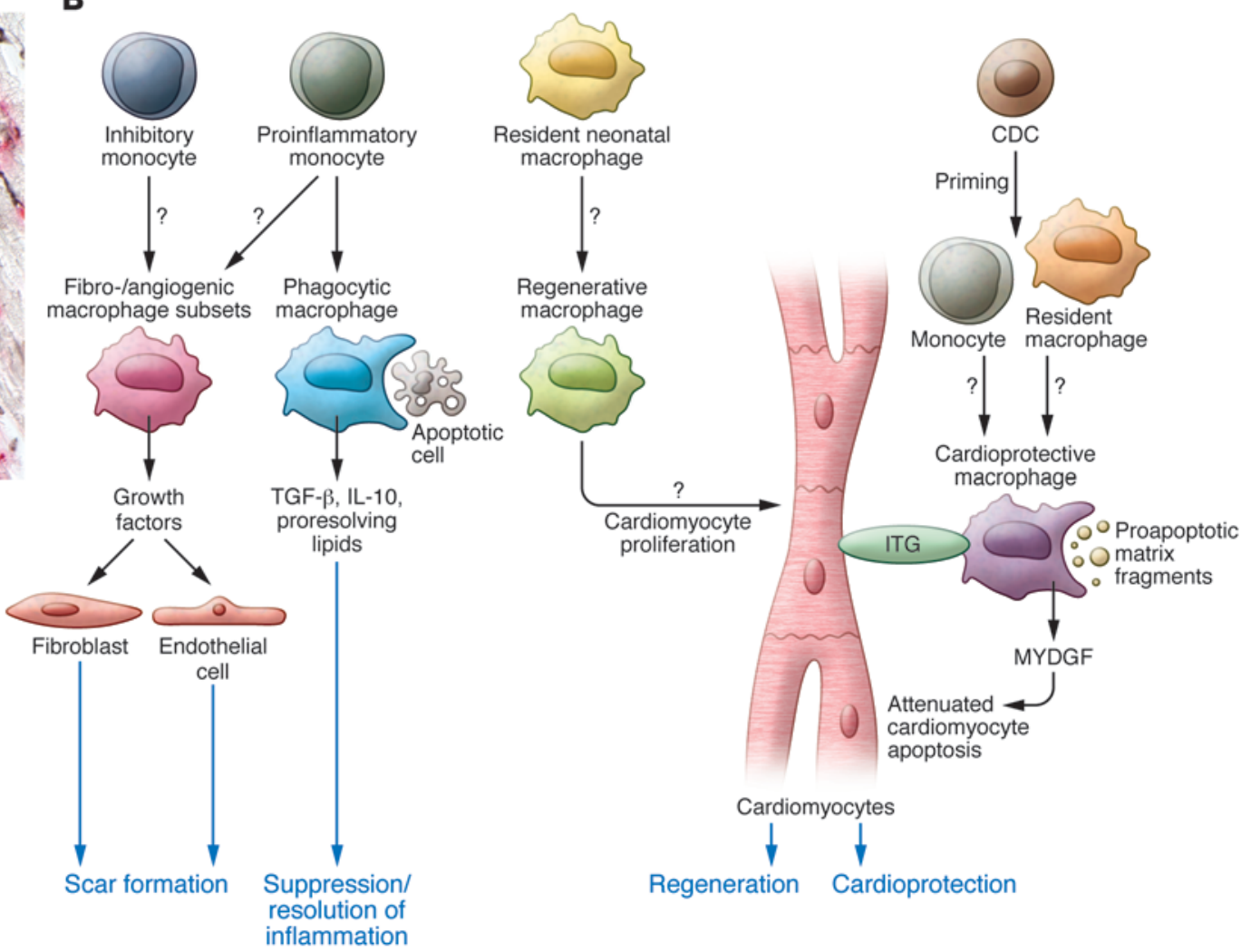

Figure 1. Abundant macrophages infiltrate the infarcted heart and can be primed to exert reparative, cardioprotective, and regenerative functions. (A) This section of reperfused canine infarct (1-hour ischemia/24-hour reperfusion) shows the presence of a large number of newly recruited myeloid cells (red) and mature macrophages (black) that are in close association with cardiomyocytes (sample prepared as previously reported, ref. 24). (B) Schematic of the multiple protective roles of infarct macrophages that have been suggested by recent experimental findings (10, 11). The adult mammalian heart contains a resident macrophage population; however, infarct macrophages are primarily derived from recruited proinflammatory and inhibitory monocyte subsets and can acquire many distinct phenotypes in response to microenvironmental cues. During the inflammatory phase of healing, macrophages may act as phagocytotic cells. Upon ingestion of apoptotic cells, macrophages release antiinflammatory mediators (such as IL-10 and TGF- $\beta$ ) that contribute to suppression of the inflammatory response. Fibrogenic and angiogenic macrophage subsets may regulate fibroblast and endothelial cell phenotypes to promote repair. Studies in neonatal mouse models of cardiac injury suggest that resident neonatal macrophages may have regenerative properties, thereby stimulating cardiomyocyte proliferation. De Couto and coworkers demonstrate that macrophages primed by CDCs protect cardiomyocytes from apoptosis. The cardioprotective actions of these macrophages may involve several distinct mechanisms, including secretion of antiapoptotic mediators (such as MYDGF), contact-dependent actions on cardiomyocyte integrin (ITC) signaling, or clearance of proapoptotic matrix fragments.

and leukemia inhibitory factor by activated macrophages has been suggested to inhibit apoptosis of hypoxic cardiomyocytes in vitro $(7,14)$. Second, contact-dependent interactions between CDC-primed macrophages and cardiomyocytes may transduce prosurvival signaling. Proapoptotic effects of macrophages on immune cells and smooth muscle cells have been reported previously and have been found to be dependent on cell-to-cell contact (15) and integrin modulation (16). Integrin signaling protects cardiomyocytes from ischemic death (17), and contact-dependent, macrophage-mediated activation of integrindependent cascades may be responsible for the protective actions. Third, as key cellular effectors of matrix remodeling, macrophages may promote prosurvival actions on cardiomyocytes by altering the cardiac extracellular matrix network. Myocardial ischemia stimulates protease activation, resulting in rapid generation of matrix fragments in the infarcted area that may exert proinflammatory and/or proapoptotic actions (18). Removal of injurious matrix fragments by activated macrophages or deposition of macrophage-derived matricellular proteins (19) may transduce prosurvival signals and protect ischemic cardiomyocytes from death.

\section{Which signals drive a cardioprotective macrophage phenotype?}

The findings by de Couto and coworkers suggest that $\mathrm{CDC}$-primed macrophages acquire a unique phenotype that is char- acterized by reduced expression of M1 macrophage markers and absence of the expected, concomitant increase in M2-related markers (9). Considering the enormous phenotypic diversity of macrophages, this observation is not surprising, as the concept of M1/M2 macrophage polarization is derived from in vitro studies and does not reflect the more nuanced phenotypes observed in vivo. The study by de Couto et al. provides limited information on the CDC-derived molecular signals that are responsible for the generation of the cardioprotective macrophages; however, the intriguing findings of these authors generate several important questions. Is the acquisition of a cardioprotective macrophage phenotype driven by secreted mediators or by exosomes 
released by CDCs? Is priming dependent on contact interactions between CDCs and macrophages? Do CDCs promote recruitment or mobilization of unique mononuclear cell subsets? Are the effects of CDCs on macrophage phenotype shared by other cell types? Answering these questions will be crucial for designing strategies for therapeutic manipulation of macrophages to protect the infarcted heart.

\section{Macrophages in cardiac repair and regeneration}

The protective actions of infarct macrophages are not limited to prosurvival effects on cardiomyocytes. Experimental evidence also suggests that macrophage subsets play a crucial role in regulating all aspects of the reparative response following infarction (20, 21). During the inflammatory phase of infarct healing, macrophages with potent phagocytotic properties clear the infarct of dead cells and matrix debris, and distinct macrophage subsets may contribute to suppression and resolution of inflammation after infarction. Upon ingestion of apoptotic cells, macrophages release antiinflammatory cytokines that may restrain inflammatory injury, attenuating adverse cardiac remodeling (22). Specialized macrophage subpopulations may also promote scar formation and angiogenesis in the infarct. Due to their abundance and phenotypic plasticity, macrophages are ideally suited to orchestrate the reparative response following infarction; however, the molecular pathways involved in generation of macrophage subsets with distinct functional properties remain poorly understood.

Recent studies suggest that macrophage subpopulations are capable of activating a regenerative program. For example, Porrello and coworkers showed that the injured heart can be remuscularized in neonatal animals following apical resection and that this regenerative capacity is lost at 7 days of age (23). Macrophage depletion abrogated remuscularization of the infarcted neonatal heart, suggesting that the unique phenotypic characteristics of neonatal macrophages may be critical for activation of a regenerative response (10). Moreover, diphtheria toxin receptor-based genetic ablation of cardiomyocytes in neonatal mice resulted in expansion of embryonic-derived cardiac macrophages that stimulated cardiomyocyte proliferation (11). In contrast, proinflammatory monocytes were recruited to the heart and inhibited the regenerative response following cardiomyocyte ablation in adult mice. Taken together, these studies suggest that cardiac macrophages coordinate myocardial regeneration; however, the molecular signals that drive macrophages toward a regenerative phenotype remain unknown. Moreover, a neonatal macrophage phenotype may not be sufficient to induce remuscularization, as formation of new myocardium may also require phenotypic plasticity of other myocardial cell types.

\section{Macrophage omnipotence: myth or reality?}

Over the last two years, our understanding of cardiac macrophages in homeostasis and disease has evolved. Emerging evidence suggests that, in the infarcted heart, macrophages have almost unlimited potential and can be primed to activate prosurvival, reparative, and regenerative responses $(10,11)$. It has been suggested that distinct macrophage subpopulations protect ischemic cardiomyocytes from death, regulate inflammation following injury, stimulate fibroblasts and vascular cells, and orchestrate remuscularization of the infarct (11). Considering their abundance, diversity, and phenotypic plasticity, macrophages are attractive candidates for serving as cellular effectors in tissue injury and repair. Manipulation of macrophage phenotypes could be exploited therapeutically to improve outcome in myocardial infarction. Unfortunately, our current knowledge of macrophage biology has substantial limitations, and conclusions about the role of macrophages in the heart following myocardial infarction are based on nonselective experimental strategies that target all monocytes and macrophages, with limited associative data on the contribution of specific macrophage subsets. Moreover, the molecular cascades involved in driving macrophages toward specific phenotypic profiles are poorly understood. Importantly, most of the evidence is derived from mouse models, and the data on phenotype and functions of human macrophages in homeostasis and disease are sparse. Despite these limitations, the recent emergence of exciting experimental evidence has placed macrophages in the center stage of cardiac pathology.

\section{Acknowledgments}

Nikolaos G. Frangogiannis' laboratory is supported by NIH grants R01 HL76246 and R01 HL85440.

Address correspondence to: Nikolaos G. Frangogiannis, Wilf Family Cardiovascular Research Institute, Albert Einstein College of Medicine, 1300 Morris Park Avenue, Forchheimer G46B, Bronx, New York 10461, USA. Phone: 718.430.3546; E-mail: nikolaos.frangogiannis@einstein.yu.edu.

1. Epelman S, et al. Embryonic and adult-derived resident cardiac macrophages are maintained through distinct mechanisms at steady state and during inflammation. Immunity. 2014;40(1):91-104.

2. Heidt T, et al. Differential contribution of monocytes to heart macrophages in steadystate and after myocardial infarction. Circ Res. 2014;115(2):284-295.

3. Mylonas KJ, et al. The adult murine heart has a sparse, phagocytically active macrophage population that expands through monocyte recruitment adopts an 'M2' phenotype in response to Th2 immunologic challenge. Immunobiology. 2015;220(7):924-933.

4. Dewald O, et al. CCL2/Monocyte chemoattractant protein-1 regulates inflammatory responses critical to healing myocardial infarcts. Circ Res. 2005;96(8):881-889.

5. Hilgendorf I, et al. Ly-6Chigh monocytes depend on Nr4a1 to balance both inflammatory and reparative phases in the infarcted myocardium. Circ Res. 2014;114(10):1611-1622.

6. Simms MG, Walley KR. Activated macrophages decrease rat cardiac myocyte contractility: importance of ICAM-1-dependent adhesion. Am JPhysiol. 1999;277(1 pt 2):H253-H260.

7. Trial J, Rossen RD, Rubio J, Knowlton AA. Inflammation and ischemia: macrophages activated by fibronectin fragments enhance the survival of injured cardiac myocytes. Exp Biol Med. 2004;229(6):538-545.

8. Marban E. Breakthroughs in cell therapy for heart disease: focus on cardiosphere-derived cells. Mayo Clin Proc. 2014;89(6):850-858.

9. de Couto $\mathrm{G}$, et al. Macrophages mediate cardioprotective cellular postconditioning in acute myocardial infarction. JClin Invest. 2015;125(8):3147-3162.

10. Aurora AB, et al. Macrophages are required for neonatal heart regeneration. J Clin Invest. 2014;124(3):1382-1392.

11. Lavine KJ, et al. Distinct macrophage lineages contribute to disparate patterns of cardiac recovery and remodeling in the neonatal and adult heart. Proc Natl Acad Sci U S A. 2014;111(45):16029-16034.

12. Reimer KA, Lowe JE, Rasmussen MM, Jennings 
RB. The wavefront phenomenon of ischemic cell death. 1. Myocardial infarct size vs duration of coronary occlusion in dogs. Circulation. 1977;56(5):786-794.

13. Korf-Klingebiel M, et al. Myeloid-derived growth factor (C19orf10) mediates cardiac repair following myocardial infarction. Nat Med. 2015;21(2):140-149.

14. Dhingra S, Sharma AK, Arora RC, Slezak J, Singal PK. IL-10 attenuates TNF- $\alpha$-induced NFאB pathway activation and cardiomyocyte apoptosis. Cardiovasc Res. 2009;82(1):59-66.

15. Zen K, Masuda J, Ogata J. Monocyte-derived macrophages prime peripheral $\mathrm{T}$ cells to undergo apoptosis by cell-cell contact via ICAM-1/

LFA-1-dependent mechanism. Immunobiology. 1996;195(3):323-333.
16. Meszaros AJ, Reichner JS, Albina JE. Macrophage-induced neutrophil apoptosis. JImmunol. 2000;165(1):435-441.

17. Okada $\mathrm{H}$, et al. Integrins protect cardiomyocytes from ischemia/reperfusion injury. J Clin Invest. 2013;123(10):4294-4308.

18. Dobaczewski M, Gonzalez-Quesada C, Frangogiannis NG. The extracellular matrix as a modulator of the inflammatory and reparative response following myocardial infarction. $\mathrm{J} \mathrm{Mol}$ Cell Cardiol. 2010;48(3):504-511.

19. Frangogiannis NG. Matricellular proteins in cardiac adaptation and disease. Physiol Rev. 2012;92(2):635-688.

20. Frantz S, et al. Monocytes/macrophages prevent healing defects and left ventricular thrombus formation after myocardial infarction. FASEB J.
2013;27(3):871-881.

21. Dutta P, Nahrendorf M. Monocytes in myocardial infarction. Arterioscler Thromb Vasc Biol. 2015;35(5):1066-1070.

22. Wan E, et al. Enhanced efferocytosis of apoptotic cardiomyocytes through myeloid-epithelialreproductive tyrosine kinase links acute inflammation resolution to cardiac repair after infarction. Circ Res. 2013;113(8):1004-1012.

23. Porrello ER, et al. Transient regenerative potential of the neonatal mouse heart. Science. 2011;331(6020):1078-1080.

24. Frangogiannis NG, et al. MCSF expression is induced in healing myocardial infarcts and may regulate monocyte and endothelial cell phenotype. Am J Physiol Heart Circ Physiol. 2003;285(2):H483-H492. 